\title{
Application of Rehabilitation Therapy in Pulmonary Infection of Stroke Patients
}

\author{
SHUMEI LI* \\ The General Ward, The Third Affiliated Hospital of Qiqihar University, No.2 Changqian Road, Tiefeng District, Qiqihar City, \\ Heilongjiang Province, China
}

\section{Li et al.: Rehabilitation therapy in pulmonary infection}

\begin{abstract}
Clinical efficacy of meridian-based massage as a rehabilitation therapy for pulmonary infection in stroke patients is explored, so as to provide new ideas and methods for the prevention and rehabilitation of stroke-associated pneumonia. Forty-six patients diagnosed as stroke complicated with pulmonary infection in The Third Affiliated Hospital of Qiqihar University are randomly divided into experimental group and control group, with 23 cases in each group. The control group is treated with medication and routine rehabilitation therapy, while the experimental group is treated with meridian massage on the basis of the control group. The results show that after $12 \mathrm{~d}$ of intervention, the pulmonary infection score of the two groups is lower than that before treatment, and the experimental group is obviously due to the control group $(\mathbf{p}<\mathbf{0 . 0 5})$. The total effective rate is $91.3 \%$ in the experimental group, which is significantly higher than $65.2 \%$ in the control group $(p<0.05)$. Therefore, massage along meridians is effective in treating stroke-associated pneumonia. It can improve the level of interleukin 4 in serum of patients with stroke related pneumonia and the ability of autoimmune regulation. It can improve the total effective rate of treatment, and effectively control the symptoms and signs of pulmonary infection. It can be used as an effective rehabilitation treatment for patients with stroke-related pneumonia. Since only $12 \mathrm{~d}$ of observation and treatment are carried out in this study, the prognosis is more one-sided, which cannot achieve a systematic and comprehensive evaluation of the treatment effect. In the future, the analysis of therapeutic effect should be more detailed.
\end{abstract}

Key words: Stroke, rehabilitation therapy, pulmonary infection, curative effect observation

Cerebral infarction, also known as ischemic stroke, is a common central nervous system injury disease. Epidemiological data show that about $21 \%-65 \%$ of acute stroke patients can be infected, and the incidence of pulmonary infection is $10.22 \%$. It is the primary reason for increasing the mortality and disability rate of post stroke patients ${ }^{[1]}$. Moreover, with the acceleration of life rhythm, overwork has become a common phenomenon, coupled with unhealthy lifestyle, leading to increased incidence of stroke, hypertension, diabetes, coronary atherosclerosis, hard heart disease and other diseases. The annual average number of hospitalized people is increasing and people have an increasing chance of hospital infection ${ }^{[2]}$. In 2003, Hilker et al. called the pneumonia of stroke patients Strokeassociated pneumonia (SAP), emphasizing that the occurrence, development, prognosis of such pneumonia is closely related to the dysfunction of the body after stroke, in order to emphasize that the occurrence of such pneumonia is fundamentally different from our previous understanding ${ }^{[3]}$. Therefore, how to prevent and treat stroke associated pneumonia has become a hot topic in clinical research. There are many risk factors for SAP. In recent years, more and more literatures report that Stroke Induced Immunodepression Syndrome (SIDS) is an important factor in the occurrence and development of $\mathrm{SAP}^{[4]}$. Apoplexy induced immunosuppression can induce a wide range of lymphocyte apoptosis, mainly B cells, T cells and macrophages. T helper (Th) cells Th1 and Th2 can produce cytokines, resulting in a reduction in the number of immune cells, function and levels of interferon, interleukin and other factors, leading to immune response impotence, weakening the body's effective defensive capacity, and increasing lung infection ${ }^{[5,6]}$. The incidence of stroke ultimately affects the prognosis of stroke ${ }^{[7]}$. With the in-depth research and development of traditional medicine, traditional Chinese medicine (TCM), acupuncture and other treatment methods gradually highlight the advantages 
of high efficacy and low toxicity and side effects. The finger point massage along meridians has the function of dredging meridians and collaterals, adjusting viscera function and enhancing disease resistance. Through the transmission of meridians and collaterals, massage along meridians can dredge meridians, balance yin and yang, regulate viscera, qi and blood, improve the pathological state of bloodstream, promote the recovery of nerve function, and improve microcirculation and nerve regeneration. Meridian massage is widely used in rehabilitation after stroke, sprain and fall, acute and chronic pain, cosmetology, health care etc. ${ }^{[8-10]}$. The therapeutic effect of modern medicine and TCM on stroke patients with pulmonary infection will be analyzed in this study, the clinical efficacy of meridian based massage as a rehabilitation treatment for stroke patients with pulmonary infection is explored, and new ideas and methods for the prevention and rehabilitation of SAP are provided, so as to benefit the majority of patients. Forty-six patients with stroke complicated with pulmonary infection are selected from April 2015 to December 2018 in The Third Affiliated Hospital of Qiqihar University. The subjects are divided into experimental group and control group with 23 cases in each group by random control table. The informed consent signed by the patients or their family members was obtained, and this study was approved by the medical ethics committee of The Third Affiliated Hospital of Qiqihar University. There is no statistical difference between the two groups $(\mathrm{p}>0.05)$ in terms of gender, age, course of disease and other general data $(p>0.05)$ as shown in Table 1. Inclusion criteria: Firstly, patients meet the clinical diagnostic criteria of traditional Chinese and Western medicine for cerebral infarction. Secondly, patients meet the clinical diagnostic criteria for stroke associated pneumonia. Thirdly, the incidence of SAP is within $7 \mathrm{~d}$. Fourthly, the patient is clear-minded, not allergic. Fifthly, family members or patients voluntarily sign informed consent to accept the study. Exclusion criteria: Firstly, patients have pneumonia after non-stroke. Secondly, patients have cerebral hernia, intracranial hypertension, severe heart, liver, kidney and other functional damage. Thirdly, patients have tumors, autoimmune, and mental

TABLE 1: GENERAL COMPARISON OF TWO GROUPS OF PATIENTS ( $\mathrm{N}=23$ )

\begin{tabular}{llll}
\hline Group & $\begin{array}{l}\text { Male/Female } \\
\text { (cases) }\end{array}$ & Age $(y)$ & $\begin{array}{l}\text { Course of } \\
\text { Disease(d) }\end{array}$ \\
\hline $\begin{array}{l}\text { Experimental } \\
\text { group }\end{array}$ & $13 / 10$ & $60.18 \pm 5.61$ & $6.28 \pm 2.21$ \\
Control group & $14 / 9$ & $59.85 \pm 5.49$ & $6.39 \pm 2.40$ \\
\hline
\end{tabular}

illness. Fourthly, patients have skin acupoints ulcer, scar or swelling and sclerosis, which are not suitable for compression. Two groups of patients are treated with routine methods. $1 \mathrm{~g}$ Cefoperazone Sulbactam Sodium is given, and $100 \mathrm{ml}$ saline is added. Anti-inflammatory drugs are injected intravenously every $12 \mathrm{~h}$, twice a d. Ambroxol is inhaled by atomization to dilute sputum, once every $8 \mathrm{~h}$, three times a d. For patients with hypertension and diabetes mellitus, the underlying diseases should be actively treated and blood pressure and blood sugar should be kept under control. $12 \mathrm{~d}$ is a course of treatment ${ }^{[11]}$. Rehabilitation therapy in control group: Firstly, for the ward environment, it is necessary to carry out air disinfection regularly, keep the room ventilated, fresh air, appropriate temperature and humidity and reduce the adverse stimulation of the environment. Secondly, attention should be paid to observing the patient's mind, pupil and other vital signs. Blood pressure needs to be measured regularly to judge the patient's mind. When the condition changes, it is necessary to treat it promptly. Thirdly, patients need to maintain a comfortable position. Patients with cough and chest tightness need to take semi-recumbent or semi-recumbent position and remove oral endocrine in time. When necessary, sputum aspiration is needed to keep the respiratory tract open. Fourthly, patients need to be assisted in turning over and kowtowing regularly. Patients need to be guided to master effective methods of coughing, expectoration and deep breathing. Fifthly, it is necessary to take medicine according to doctor's instructions, and do not increase or decrease the dosage or stop taking medicine at will. Chinese herbal decoction should be taken warmly. It should be separated from western medicine by $30 \mathrm{~min}$ to observe the reaction after taking medicine. Sixthly, for rehabilitation guidance, it is necessary to observe the changes of limb dysfunction and adhere to the functional exercise of healthy limbs. For patients with language function, communication should be carried out through gestures, pictures and other aids and language training should be carried out at an early stage. Rehabilitation therapy in the experimental group: On the basis of the control group, massage along meridians is added. Assessment: past history, clinical symptoms, massage site skin, pain tolerance. Location: The location of acupoints and massage methods are determined. The great vertebra is in the depression of the $7^{\text {th }}$ cervical spinous process. Feishu is on the back of the human body, under the third thoracic spinous process and 1.5 inches apart from the left and right sides. Fenglong is in the front and outside of the human leg, 8 inches above the tip of the lateral 
ankle and two transverse fingers on the front of the tibia. Cloud Gate is located in the chest, in the depression of subclavian fossa, at the inner edge of the coracoid process of scapula, and 6 inches apart from the anterior median line. Zhongfu is located in the chest, horizontal the first rib space, lateral subclavian fossa, and 6 inches apart from the anterior median line ${ }^{[12]}$. Taiyuan is located in the anterior carpal region, between the styloid process of radius and scaphoid bone, in the ulnar depression of the abductor pollicis longus tendon, the lateral tendon of the radial carpal region, and the medial tendon of the abductor pollicis longus. The thenar is located on the radial side of the middle point of the first metacarpal bone and the red-white interstitial area. Finger point: The patient is in prone position. The operator accurately selects great vertebra, Feishu and Fenglong acupoints and marks them well. With the thumb fingertip to focus on the acupoints, it needs continuous point pressure and each acupoint point is pressed for $3 \mathrm{~min}$. Patient's feeling of local acidity and distension is regarded as a criterion. The procedure is repeated 3 to 5 times at intervals of $1 \mathrm{~min}$ for a total of 10 min. Meridian massage: The patient takes a sitting position. Hand push over Taiyin and massage cream is used for massage ${ }^{[13]}$. Acupoint markers such as Yunmen, Zhongfu, Taiyuan and thenar are selected. From top to bottom, the thumb pulp is used to focus on the lungs of the hand and Taiyin. The first metacarpophalangeal joint is gently rotated and rubbed. When passing through Yunmen, Zhongfu, Taiyuan, Yujian and other acupoints, it is necessary to press down and stay for a while. Each acupoint lasts for $2 \mathrm{~min}$, alternating sides, with two times a d and $12 \mathrm{~d}$ as a course of treatment. The changes of clinical pulmonary infection score (CPIS) before and $12 \mathrm{~d}$ after treatment are observed. The levels of serum interleukin 4 (IL-4) are observed before treatment and $12 \mathrm{~d}$ after treatment. $1 \mathrm{~d}$ before treatment and $12 \mathrm{~d}$ after treatment, each group takes about $3 \mathrm{ml}$ of blood on an empty stomach for 1-2 $\mathrm{h}^{[14]}$. The blood is placed in a centrifuge and centrifuged at $3000 \mathrm{P} / \mathrm{m}$ for $15 \mathrm{~min}$. The separated serum is sucked out by a suction tube. After the serum is separated, IL-4

TABLE 2: COMPARISON OF CLINICAL EFFICACY BETWEEN THE TWO GROUPS

\begin{tabular}{lcccc}
\hline Group & $\begin{array}{c}\text { Markedly } \\
\text { effective }\end{array}$ & Effective & Invalid & $\begin{array}{c}\text { Total } \\
\text { effective } \\
\text { rate }\end{array}$ \\
\hline $\begin{array}{l}\text { Experimental } \\
\text { group }\end{array}$ & 12 & 9 & 2 & $91.3 \%$ \\
Control group & 7 & 8 & 8 & $65.2 \%$ \\
$x^{2}$ & - & - & - & 9.453 \\
p & - & - & - & $<0.05$ \\
\hline
\end{tabular}

is detected by flow cytometry reagent and flow cytometry reagent produced by Roche Diagnosis Co., Ltd. of Germany. Therapeutic evaluation criteria are as follows. Rehabilitation: normal body temperature, disappearance of clinical symptoms and signs and normal white blood cell count. Chest radiographs show absorption of inflammatory reaction in the lungs and negative culture of pathogenic bacteria in sputum. Markedly effective: normal body temperature, basic disappearance of clinical symptoms and signs and normal white blood cell count. Chest X-ray shows that most of the pulmonary inflammatory responses are absorbed. Effective: Body temperature is basically normal and clinical symptoms and signs have improved. White blood cell count is normal or higher than normal and chest X-ray shows that pulmonary inflammatory response has been absorbed. Invalid: Body temperature is basically normal or higher than normal and clinical symptoms and signs have no change or aggravation. White blood cell count is higher than normal and chest $\mathrm{X}$-ray shows no absorption of pulmonary inflammatory response $^{[15]}$. Total effective rate $=$ Cure number + Effective number/Total number $\times 100 \%$ (1) SPSS22.0 statistical software is used to deal with the data of symptomatology score before and after treatment. The counting data are expressed by $\mathrm{n} \%$ and $\chi^{2}$ test. Measurement data is expressed by $\bar{x} \pm s$ and $t$ test. The difference is statistically significant with $\mathrm{p}<0.05$. CPIS score is a scoring system that integrates clinical, radiological and microbiological criteria to assess the severity of pulmonary infection. It can be used to guide the assessment of the severity of pulmonary infection and predict the efficacy and prognosis. There are seven indicators, including body temperature, white blood cell count, tracheal secretion, oxygenation, chest X-ray, progress of lung infiltration shadow and tracheal extract culture. Before treatment, the CPIS scores of the experimental group and the control group are $7.42 \pm 1.09$ and $7.56 \pm 1.18$ respectively. After $12 \mathrm{~d}$ of treatment, the CPIS scores of the experimental group and the control group are $2.25 \pm 0.56$ and 3.96 \pm 0.84 respectively. The CPIS scores of the two groups are significantly lower than those before treatment and the CPIS scores of the experimental group and the control group are significantly lower than those of the control group after treatment $(p<0.05)$. The difference is statistically significant $(\mathrm{p}<0.05)$, as shown in fig. 1. Activated human T cells are divided into Th1 (Interferons (IFN)-a) and RNA ligase 2 (rnl2) (lL-4) according to the different secreted cytokines. The proinflammatory cytokines increases in cerebral ischemia. At the same time, it is found that by stimulating 
the sympathetic adrenal medullary system, rapid and lasting suppression of cellular immune function is induced. It is characterized by decreased monocyte activity, decreased $\mathrm{T}$ cell count and Thlflla 2 transformation, which reduces the production of IL-4 and causes spontaneous systemic bacterial infection and pneumonia. Before treatment, the serum levels of IL-4 in the experimental group and the control group are $19.87 \pm 3.94$ and $20.04 \pm 4.11$ respectively. After $12 \mathrm{~d}$ of treatment, the serum levels of IL-4 in the experimental group and the control group are $94.21 \pm 10.89$ and $82.78 \pm 9.76$ respectively. The level of serum IL-4 in the two groups is significantly higher than that before treatment, and that in the experimental group is higher than that in the control group $(\mathrm{p}<0.05)$. The difference is statistically significant ( $p<0.05)$, as shown in fig. 2 . In this study, Feishu point is selected for point massage and Feishu belongs to Back-shu point. Back-shu point is close to the viscera, which has the function of tonifying lung qi, tonifying deficiency and clearing

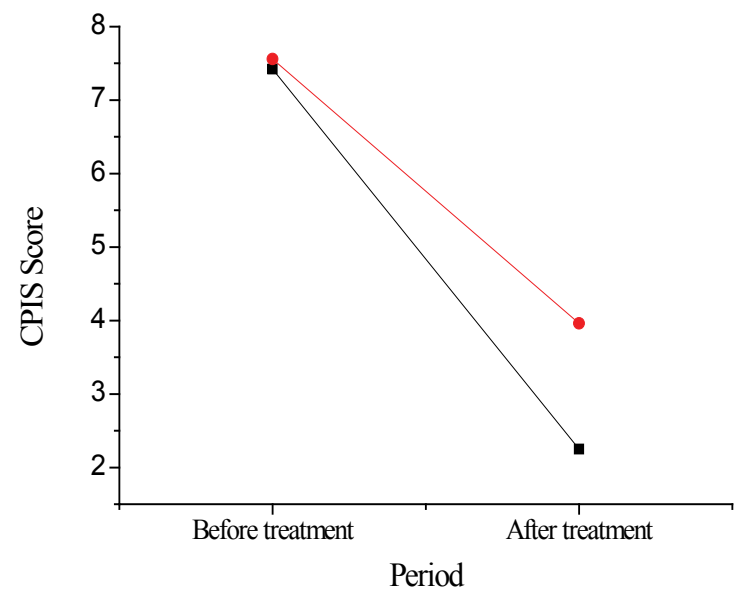

Fig. 1: CPIS score of two groups of patients Experimental group (-・), control group (

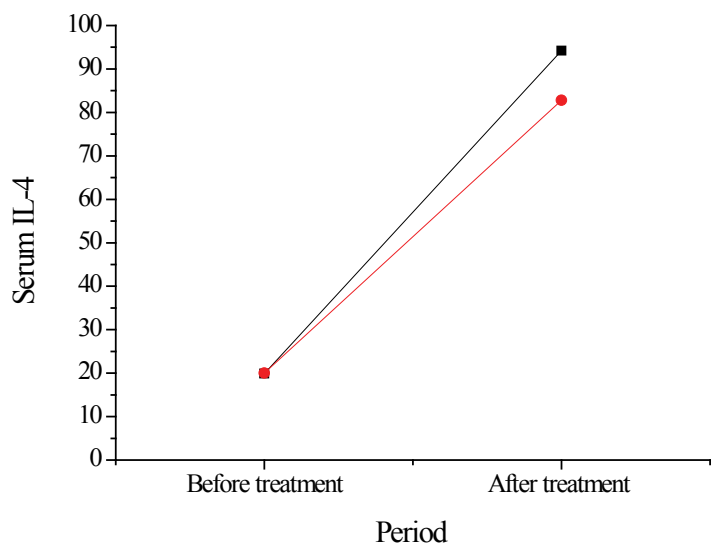

Fig. 2: Serum IL-4 levels in two groups Experimental group (-"), control group (-"-) heat, so it can play a very good role in regulating the adjacent viscera. Because of its rich nerve endings and sensitive acupoint response, it can fully stimulate the human body's meridian conduction and nerve body fluid regulation, thus preventing and treating diseases. After $12 \mathrm{~d}$ of treatment, the effective rate of the experimental group is $91.3 \%$ and that of the control group is $65.2 \%$. The experimental group is significantly higher than that of the control group $(\mathrm{p}<0.05)$ and the difference is statistically significant, as shown in Table 2. Meridian massage intervention therapy can greatly reduce the incidence of complications of stroke related pneumonia in stroke patients and win time for patients to carry out later rehabilitation exercises. It plays an important role in the prevention and treatment of multiple organ failure caused by middle aged and elderly stroke patients. Meridian massage, as a commonly used clinical rehabilitation treatment technique of TCM, is guided by the theoretical basis of TCM, based on viscera theory. Meridian theory is taken as the core. Through stimulating specific parts of the human body and regulating qi and blood, the functions of corresponding organs are stimulated to strengthen the body, dispel pathogens, prevent diseases and protect diseases. At present, with the vigorous development of the cause of TCM, the promotion and application of rehabilitation treatment technology of TCM has been gradually promoted to grass-roots hospitals and gradually extended to communities and families. Because of the convenience, effectiveness and safety of the operation technology of rehabilitation therapy with Chinese characteristics, its efficacy has been widely accepted and recognized and it plays an important role in the fields of chronic diseases, geriatric diseases and preventive treatment. This study shows that massage along meridians can significantly reduce airway smooth muscle spasm and inflammatory injury of airway mucosa caused by inflammatory mediators, and reduces airway hyper responsiveness. It can also inhibit the synthesis and secretion of tumor necrosis factor- $\alpha$ (TNF- $\alpha$ ), thus blocking the release of inflammatory mediators, alleviating airway inflammation, alleviating the spasm of tracheal smooth muscle and improving immune indexes such as $1 \mathrm{~L}-4$. Therefore, it can be seen that the method of selecting Feishu point as the main point and massaging along the meridians of hand, Taiyin and lung is effective in preventing and treating stroke-related pneumonia. Because of the small number of samples observed in this study, only the patients with cerebral infarction complicated with SAP are observed for $12 \mathrm{~d}$ as a course of treatment. If the patients with 
cerebral infarction complicated with SAP can be included in the observation in the future, the observation time can be prolonged and more detailed determination of inflammatory factors can be made, it might be possible to excavate more effective means of regulating post-stroke immune suppression from the rehabilitation treatment of TCM and prevent and treat stroke related pneumonia.

\section{REFERENCES}

1. Smithard DG. Dysphagia management and stroke units. Curr Phys Med Rehabil Rep 2016;4(4):287-94.

2. Guillén-Solà $A$, Messagi Sartor M, Bofill Soler N, Duarte E, Barrera MC, Marco E. Respiratory muscle strength training and neuromuscular electrical stimulation in subacute dysphagic stroke patients: a randomized controlled trial. Clin Rehabil 2017;31(6):761-71.

3. Hamada S, Yamaguchi H, Hara H. Does sensory transcutaneous electrical stimulation prevent pneumonia in the acute stage of stroke? A preliminary study. Int J Rehabil Res 2017; 40(1):94-6.

4. Shi GM, Zhang YD, Geng C, Zhang YQ, Pan XD, Liu YK, et al. Profile and 1-year outcome of ischemic stroke in East China: Nanjing first Hospital stroke Registry. J Stroke Cerebrovasc Dis 2016;25(1):49-56.

5. Sui X. Inhibition of the NF- $\kappa B$ signaling pathway on endothelial cell function and angiogenesis in mice with acute cerebral infarction. J Biol Regul Homeost Agents 2019;33(2):375-84.

6. Zheng Y, Song T, Zhang L, Wei N. Immunomodulatory effects of T helper 17 cells and regulatory T cells on cerebral ischemia. J Biol Regul Homeost Agents 2018;32(1):29-35.

7. Civelek GM, Atalay A, Turhan N. Medical complications experienced by first-time ischemic stroke patients during inpatient, tertiary level stroke rehabilitation. J Phys Ther Sci 2016;28(2):382-91.

8. Yuan MZ, Li F, Fang Q, Wang W, Peng JJ, Qin DY, et al. Research on the cause of death for severe stroke patients. J
Clin Nurs 2018;27(1-2):450-60.

9. Larsen T, Lee A, Brooks D, Michieli S, Robson M, Veens J, et al. Effect of early mobility as a physiotherapy treatment for pneumonia: a systematic review and meta-analysis. Physiother Can 2019;71(1):82-9.

10. Rosa M, De Lucia S, Rinaldi VE, Le Gal J, Desmarest M, Veropalumbo $\mathrm{C}$, et al. Paediatric arterial ischemic stroke: acute management, recent advances and remaining issues. Ital J Pediatr 2015;41(1):1-2.

11. Ferdinand P, Roffe C. Hypoxia after stroke: a review of experimental and clinical evidence. Exp Transl Stroke Med 2016;8(1):9.

12. Paz AL, Doniz LG, García SO, Canosa JL, Couto CM. Respiratory muscle strength in chronic stroke survivors and its relation with the 6-minute walk test. Arch Phys Med Rehab 2016;97(2):266-72.

13. De Menezes KK, Nascimento LR, Polese JC, Ada L, TeixeiraSalmela LF. Effect of high-intensity home-based respiratory muscle training on strength of respiratory muscles following a stroke: a protocol for a randomized controlled trial. Braz $\mathrm{J}$ Phys Ther 2017;21(5):372-7.

14. Cohen DL, Roffe C, Beavan J, Blackett B, Fairfield CA, Hamdy $\mathrm{S}$, et al. Post-stroke dysphagia: a review and design considerations for future trials. Int J Stroke 2016;11(4):399411.

15. Chang KC, Hung JW, Lee HC, Yen CL, Wu CY, Yang CL, et al. Rehabilitation Reduced Readmission and Mortality Risks in Patients with Stroke or Transient Ischemic Attack. Medical care 2018;56(4):290-8.

This is an open access article distributed under the terms of the Creative Commons Attribution-NonCommercial-ShareAlike 3.0 License, which allows others to remix, tweak, and build upon the work non-commercially, as long as the author is credited and the new creations are licensed under the identical terms

This article was originally published in a special issue, "Trends in Therapeutic Management of Various Clinical Conditions II" Indian J Pharm Sci 2021:83(2)Spl issue;51-55 